\title{
Relativistic corrections to bound-state energies for two-fermion systems
}

\author{
F. Gesztesy* \\ Zentrum für Interdisziplinäre Forschung, Universität Bielefeld, \\ D-4800 Bielefeld 1, Federal Republic of Germany
}

\author{
H. Grosse G $^{\dagger}$ \\ CERN CH-1211 Geneva, 23
}

\author{
B. Thaller \\ Institut für Theoretische Physik, Universität Graz, A-8010 Graz, Austria
}

(Received 14 May 1984)

\begin{abstract}
Holomorphy of the Breit-Fermi resolvent around the nonrelativistic limit is used to derive a new method for calculating first-order relativistic corrections to bound-state energies.
\end{abstract}

\section{INTRODUCTION}

The problem we are discussing is a rather old one and simple to state. Start with the Dirac operator with static external fields. Under mild assumptions on the potentials the spectrum will consist of continuous parts with a gap around energy zero and possibly bound states in the gap. The problem now is how to get information on the bound-state energies. In the absence of a variational principle, the most well-known procedure consists in formally expanding the Dirac operator for inverse powers of the velocity of light $c$. As a first step this Foldy-Wouthuysen procedure $^{1}$ gives highly singular operators. In a second step one applies first-order perturbation theory which, however, is completely unjustified. Although we have been able to give an interpretation to the second step in terms of spectral concentration, ${ }^{2,3}$ one should try to avoid the above-mentioned procedure completely. For the oneparticle case we succeeded to prove holomorphy of the Dirac resolvent in $c^{-1}$. From the explicit expansion of the resolvent we then extracted corrections to bound-state energies of the Pauli operator., ${ }^{4,5}$

In this paper we shall apply our method to the twoparticle case. To do so, we have to have a Hamilton operator. In principle, one should start from a relativistic wave equation (Bethe-Salpeter equation or some other bound-state equations) and try to obtain a Hamiltonian by an instantaneous limit. Concerning this (certainly nonunique) step, we have nothing to say (except for some comments at the end of the paper). Here we assume that these steps can be done. For QED this yields the so-called Breit-Fermi Hamiltonian. $^{6-8}$ At this point we should add a warning: In case the potential is determined up to a certain order in a coupling constant (or in $1 / c^{2}$ ) it does not make sense to continue our procedure to higher orders in these parameters. We also remark that our procedure works for local as well as nonlocal interactions.

In order to clarify the above remarks, let us discuss the Hamiltonian for the positronium atom. Let $\widetilde{H}(c)$ $=H_{0}(c)+V$ with

$$
\begin{aligned}
H_{0}(c)= & \left(c \vec{\alpha}_{1} \cdot \overrightarrow{\mathrm{p}}+\beta_{1} m_{1} c^{2}\right) \otimes \mathbb{1}_{2} \\
& +\mathbb{1}_{1} \otimes\left(-c \vec{\alpha}_{2} \cdot \overrightarrow{\mathrm{p}}+\beta_{2} m_{2} c^{2}\right) .
\end{aligned}
$$

$H_{0}(c)$ represents the free Hamiltonian of two Dirac particles in the center-of-mass frame and acts in

$$
L^{2}\left(\mathrm{R}^{3}\right) \otimes \mathrm{C}^{4} \otimes \mathrm{C}^{4} \text {. }
$$

$\vec{\alpha}_{1}$ and $\beta_{1}\left(\vec{\alpha}_{2}\right.$ and $\left.\beta_{2}\right)$ are Dirac matrices for particle one (two). $\mathbb{1}_{1}$ and $\mathbb{1}_{2}$ are $4 \times 4$ unit matrices.

The main question concerns the potential $V$. Take, for instance,

$$
\begin{aligned}
V(\overrightarrow{\mathrm{x}})= & v(r) \mathbb{1}_{1} \otimes \mathbb{1}_{2}+w_{1}(r) \vec{\alpha}_{1} \otimes \vec{\alpha}_{2} \\
& +w_{2}(r)\left(\vec{\alpha}_{1} \cdot \hat{x}\right) \otimes\left(\vec{\alpha}_{2} \cdot \hat{x}\right),
\end{aligned}
$$

where $\overrightarrow{\mathbf{x}}$ denotes the relative coordinate, $r=|\overrightarrow{\mathbf{x}}|$ and $\hat{x}=\vec{x} / r$; then for the special case

$$
v(r)=-2 w_{1}(r)=-2 w_{2}(r)=-\frac{e^{2}}{r},
$$

Eqs. (1.1) to (1.3) represent the Breit-Fermi operator and the question of a nonrelativistic expansion of bound-state energies can be posed.

We shall also be concerned with more general interactions than (1.2) and (1.3), which are of interest for quarkonium spectroscopy. The general ansatz we take for $V$ is of the form

$$
V=\left(\begin{array}{cccc}
v_{+} & 0 & 0 & w_{+} \\
0 & v_{-} & w_{-} & 0 \\
0 & w_{-} & v_{-} & 0 \\
w_{+} & 0 & 0 & v_{+}
\end{array}\right),
$$

where we have used a $16 \times 16$ matrix notation and each entry of the matrix in (1.4) represents a $4 \times 4$ matrix. It can be shown that special cases of (1.4) will account for general interactions coming from scalar, vector, pseudoscalar, axial-vector, and tensor interactions, although we shall concentrate only on the two first mentioned. 
There is a long list of publications concerned with relativistic corrections to bound-state energies and decay parameters (cf. e.g., Refs. 9-12 and the references therein). It is worth mentioning that for a scalar interaction there exist three different proposals for the correction terms. ${ }^{13-15}$ We shall see that our procedure gives a result in agreement with Ref. 15. For a determination of the structure of spin-dependent forces in QCD see Refs. 16--18.

We shall be dealing only with two-body systems; there are additional problems for the many-body case. ${ }^{19}$ In Sec. II we shall explain our method for interactions like (1.4). Section III is devoted to a discussion of special interactions and to a comparison with results in the literature.

\section{POSITRONIUM}

We use the representation

$$
\vec{\alpha}=\left(\begin{array}{ll}
0 & \vec{\sigma} \\
\vec{\sigma} & 0
\end{array}\right), \quad \beta=\left(\begin{array}{cc}
1 & 0 \\
0 & -\mathbb{1}
\end{array}\right),
$$

then $H_{0}$ can be written in a $16 \times 16$ matrix form:

$$
\begin{aligned}
h_{0}(c) & =H_{0}(c)-m_{1} c^{2}-m_{2} c^{2} \\
& =\left(\begin{array}{cc}
0 & c A_{1} \\
c A_{1} & -2 m_{1} c^{2}
\end{array}\right) \otimes \mathbb{1}_{2}+\mathbb{1}_{1} \otimes\left[\begin{array}{cc}
0 & c A_{2} \\
c A_{2} & -2 m_{2} c^{2}
\end{array}\right)
\end{aligned}
$$

where we introduced the notation $A_{1}=\vec{\sigma}_{1} \cdot \overrightarrow{\mathrm{p}}, A_{2}$ $=-\vec{\sigma}_{2} \cdot \overrightarrow{\mathrm{p}}$ and subtracted rest energies from $H_{0}(c)$. Next define the free resolvent $R_{z}^{0}(c)$ :

$$
R_{z}^{0}(c)=\left[h_{0}(c)-z\right]^{-1}, \quad z \in \mathrm{C} \backslash \mathrm{R} .
$$

Since all entries in $h_{0}(c)$ commute with each other $h_{0}(c)-z$ can be inverted in a straightforward calculation. For notational simplicity we state the result in the special case $m_{1}=m_{2}=\frac{1}{2}$ :

$$
R_{z}^{0}(c)=\left[2 p^{2}-z-\frac{z^{2}}{2 c^{2}}\right]^{-1}\left[P_{+}^{1} \otimes P_{+}^{2}+\frac{1}{c^{2}+z} M(c)\right)
$$

where we introduced the projection operators $P_{+}^{1,2}=\left(1+\beta^{1,2}\right) / 2$, and the symmetric matrix $M(c)$ in a $4 \times 4$ notation is given by

$$
\begin{array}{ll}
M_{11}=\frac{z}{2}+\frac{z^{2}}{2 c^{2}}-p^{2}, & M_{22}=z+\frac{z^{2}}{2 c^{2}}-p^{2}=M_{33} \\
M_{12}=A_{1}\left(c+\frac{z}{2 c}\right), & M_{24}=A_{2} \frac{z}{2 c} \\
M_{13}=A_{2}\left(c+\frac{z}{2 c}\right), & M_{34}=A_{1} \frac{z}{2 c}, \\
M_{14}=A_{1} A_{2}=M_{23}, & M_{44}=\frac{z}{2}+\frac{z^{2}}{2 c^{2}}-p^{2} .
\end{array}
$$

In particular in the nonrelativistic limit $c \rightarrow \infty, R_{z}^{0}(c)$ converges to the free Pauli resolvent $\left(p^{2} / 2 \mu-z\right)^{-1}$ (where $\mu^{-1}=m_{1}^{-1}+m_{2}^{-1}$ is the reduced mass) times a projection operator:

$$
\underset{c \rightarrow \infty}{\mathrm{n}-\lim _{z}} R_{z}^{0}(c)=\left(\frac{p^{2}}{2 \mu}-z\right)^{-1} P_{+}^{1} \otimes P_{+}^{2}
$$

Equations (2.4) and (2.5) clearly demonstrate that $R_{z}^{0}(c)$ is holomorphic in $c^{-1}$ around the nonrelativistic limit $c^{-1}=0$.

But one notices a difference between the one-particle and the two-particle case. For the one-particle case even after multiplying the free resolvent with potentials which are relatively bounded with respect to $\vec{\alpha} \cdot \vec{p}$ norm convergence was obtained. ${ }^{4 ; 5}$ Since this is not the case here we shall assume that $V$ is a bounded operator in order to avoid problems of self-adjointness. Thus, we assume that $v_{ \pm}$and $w_{ \pm}$are essentially bounded from now on.

The main idea of our approach consists in discussing the resolvent of $H(c)=h_{0}(c)+V$ (which is a bounded operator),

$$
R_{z}(c)=[H(c)-z]^{-1}, \quad z \in \mathrm{C} \backslash \mathrm{R},
$$

instead of dealing with the unbounded Hamiltonian $H(c)$. Next we shall extract in a two-step procedure parts of $H(c)$ which dominate in the limit $c \rightarrow \infty$ and write $H(c)-z=D(c)+E(c)$ with

$$
\begin{aligned}
D(c) & =\left(\begin{array}{cccc}
-z & c A_{1} & c A_{2} & 0 \\
c A_{1} & -2 m_{1} c^{2} & 0 & 0 \\
c A_{2} & 0 & -2 m_{2} c^{2} & 0 \\
0 & 0 & 0 & -2\left(m_{1}+m_{2}\right) c^{2}
\end{array}\right) \\
E(c) & =\left(\begin{array}{cccc}
v_{+} & 0 & 0 & w_{+} \\
0 & v_{-}-z & w_{-} & c A_{2} \\
0 & w_{-} & v_{-}-z & c A_{1} \\
w_{+} & c A_{2} & c A_{1} & v_{+}-z
\end{array}\right) .
\end{aligned}
$$

Since all elements of $D(c)$ commute it is trivial to invert $D(c)$. Making use of the identity

$$
(D+E)^{-1}=\left(1+D^{-1} E\right)^{-1} D^{-1}
$$

allows, after multiplying out $D^{-1}(c)$ and $E(c)$, to conclude that in the limit $c \rightarrow \infty, R_{z}(c)$ tends to the (interacting) Pauli resolvent in norm:

$$
\mathrm{n}_{c \rightarrow \infty} R_{z}(c)=\left(\frac{p^{2}}{2 \mu}+v_{+}-z\right)^{-1} P_{+}^{1} \otimes P_{+}^{2}, \quad z \in \mathrm{C} \backslash \mathrm{R} .
$$

Moreover, following the derivation of Eq. (2.15) in Ref. 5, the explicit form of $D^{-1}(c)$ finally yields our first main result.

Let $H(c)=h_{0}(c)+V, V$ bounded, and $z \in C \backslash R$. Then $R(z)=[H(c)-z]^{-1}$ is holomorphic in $c^{-1}$ around $c^{-1}=0$. At first sight it might be surprising that the resolvent is analytic as a function of $c^{-1}$, while the folklore results for expansions of eigenvalues yield a series in $c^{-2}$. But as in the one-particle case one can go over to a modified resolvent $\widetilde{R}_{z}(c)$, 


$$
\begin{aligned}
& \widetilde{R_{z}}(c)=B(c) R_{z}(c) B^{-1}(c) \\
& B(c)=\left(\begin{array}{llll}
1 & 0 & 0 & 0 \\
0 & c & 0 & 0 \\
0 & 0 & c & 0 \\
0 & 0 & 0 & c^{2}
\end{array}\right)
\end{aligned}
$$

and with the help of the similarity transformation $B(c)$ one observes that $\widetilde{R}_{z}(c)$ becomes holomorphic as a function of $c^{-2}$ around $c^{-2}=0$.

More explicitly we first note that $B\left(D^{-1} E\right) B^{-1}$ converges in norm to

$$
\begin{aligned}
& d=\mathrm{n}_{c \rightarrow \infty} \lim _{c \rightarrow \infty} B(c) D^{-1}(c) E(c) B^{-1}(c) \\
& =\left(\begin{array}{cccc}
\rho_{z}^{0} v_{+} & 0 & 0 & 0 \\
\rho_{z}^{0} \frac{A_{1}}{2 m_{1}} v_{+} & 0 & 0 & 0 \\
\rho_{z}^{0} \frac{A_{2}}{2 m_{2}} v_{+} & 0 & 0 & 0 \\
-\frac{w_{+}}{2\left(m_{1}+m_{2}\right)} & -\frac{A_{2}}{2\left(m_{1}+m_{2}\right)} & -\frac{A_{1}}{2\left(m_{1}+m_{2}\right)} & 0
\end{array}\right), \\
& \rho_{z}^{0}=\left[\frac{p^{2}}{2 \mu}-z\right]^{-1} .
\end{aligned}
$$

In order to get a norm-convergent expansion of $\widetilde{R}_{z}(c)$ in terms of $c^{-2}$, we apply formulas (2.9) once again and take out $1+d$ from $B\left(1+D^{-1} E\right)^{-1} B^{-1}$ :

$$
\begin{aligned}
\widetilde{R}_{z}= & {[1+d+e(c)]^{-1} \widetilde{D}-1(c) } \\
= & {\left[1+(1+d)^{-1} e(c)\right]^{-1}(1+d)^{-1} \widetilde{D}^{-1}(c), } \\
& \widetilde{D}(c)=B(c) D(c) B^{-1}(c),
\end{aligned}
$$

where $e(c)$ denotes the corresponding remaining part. Explicit multiplication of the matrices in (2.13) then shows after some calculations that

$$
\begin{aligned}
& \underset{c \rightarrow \infty}{\mathrm{n}-\lim _{z}} \widetilde{R_{z}}(c)=\left(\begin{array}{cccc}
\rho_{z} & 0 & 0 & 0 \\
\frac{A_{1}}{2 m_{1}} \rho_{z} & 0 & 0 & 0 \\
\frac{A_{2}}{2 m_{2}} \rho_{z} & 0 & 0 & 0 \\
W \cdot \rho_{z} & 0 & 0 & 0
\end{array}\right]=\widetilde{R}_{0}(z), \\
& W=\frac{w_{+}}{2\left(m_{1}+m_{2}\right)}+\frac{A_{1} A_{2}}{4 m_{1} m_{2}}, \\
& \rho_{z}=\left[\frac{p^{2}}{2 \mu}+v_{+}-z\right]^{-1},
\end{aligned}
$$

and that $\widetilde{R}_{z}(c)$ is indeed holomorphic with respect to $c^{-2}$ around the nonrelativistic limit

$$
\widetilde{R_{z}}(c)=\widetilde{R}_{0}(c)+c^{-2} \widetilde{R}_{1}(z)+O\left(c^{-4}\right) .
$$

Since $\widetilde{R}_{1}(z)$ has a quite complicated structure we only quote those matrix elements that are needed for later purposes:

$$
\begin{aligned}
{\left[\widetilde{R}_{1}(z)\right]_{11}=} & -\rho_{z}\left(\frac{A_{1}}{2 m_{1}}\left(v_{-}-z\right)+\frac{A_{2}}{2 m_{2}} w_{-}\right) \frac{A_{1}}{2 m_{1}} \rho_{z} \\
& -\rho_{z}\left(\frac{A_{1}}{2 m_{1}} w_{-}+\frac{A_{2}}{2 m_{2}}\left(v_{-}-z\right)\right) \frac{A_{2}}{2 m_{2}} \rho_{z} \\
& -\rho_{z} W^{2} \rho_{z}, \\
{\left[\widetilde{R}_{1}(z)\right]_{12}=} & \rho_{z} A_{1} / 2 m_{1},\left[\widetilde{R}_{1}(z)\right]_{13}=\rho_{z} A_{2} / 2 m_{2}, \\
{\left[\widetilde{R}_{1}(z)\right]_{14}=} & 0 .
\end{aligned}
$$

In order to derive our second main results, namely, an explicit formula for first-order relativistic corrections of bound-state energies, we follow the strategy developed in Refs. 4 and 5. From the fact that

$$
\left[\widetilde{R}_{0}(z)-\zeta\right]^{-1}=\left(\begin{array}{cccc}
\left(\rho_{z}-\zeta\right)^{-1} & 0 & 0 & 0 \\
\xi^{-1} \frac{A_{1}}{2 m_{1}} \rho_{z}\left(\rho_{z}-\zeta\right)^{-1} & \xi^{-1} & 0 & 0 \\
\xi^{-1} \frac{A_{2}}{2 m_{2}} \rho_{z}\left(\rho_{z}-\zeta\right)^{-1} & 0 & \zeta^{-1} & 0 \\
\zeta^{-1} \cdot W \cdot \rho_{z}\left(\rho_{z}-\zeta\right)^{-1} & 0 & 0 & \zeta^{-1}
\end{array}\right),
$$

we infer as in Lemma 2.2 of Ref. 5 that there is a one-toone correspondence between nonzero eigenvalues of $\rho_{z}$ and $\widetilde{R}_{0}(z)$. In particular,

$$
\begin{aligned}
& \left(\frac{p^{2}}{2 \mu}+v_{+}\right) f_{0}=E_{0} f_{0}, \text { i.e., } \rho_{z} f_{0}=\left(E_{0}-z\right)^{-1} f_{0}, \\
& E_{0} \in \mathrm{R}, \quad z \in \mathrm{C} \backslash \mathrm{R},
\end{aligned}
$$

implies

$$
\left[\widetilde{R}_{0}(z)-\left(E_{0}-z\right)^{-1}\right] \Phi_{0}=0, \quad \Phi_{0}=\left(\begin{array}{c}
f_{0} \\
\frac{A_{1}}{2 m_{1}} f_{0} \\
\frac{A_{2}}{2 m_{2}} f_{0} \\
W f_{0}
\end{array}\right) .
$$

Since $\widetilde{R}_{z}(c)$ is holomorphic with respect to $c^{-2}$ around the nonrelativistic limit, analytic perturbation theory implies that eigenvalues and eigenfunctions of $\widetilde{R}_{z}(c)$ and $H(c)$ [by the similarity transformation $B(c)$ ] are also analytic with respect to $c^{-2}$. Assuming for simplicity that $E_{0}$ is a nondegenerate discrete eigenvalue of the Pauli Hamiltonian $p^{2} / 2 \mu+v_{+}$and that $E\left(c^{-2}\right)$ is the corresponding relativistic eigenvalue of $H(c)$ [with $E(0)=E_{0}$ ] then $E\left(c^{-2}\right)$ is also nondegenerate and we can expand

$$
E\left(c^{-2}\right)=E_{0}+c^{-2} E_{1}+O\left(c^{-4}\right) .
$$

To calculate $E_{1}$ explicitly we apply first-order perturbation theory to Eq. (2.15). A standard procedure for 
bounded (but nonself-adjoint) operators then leads to

$$
\begin{aligned}
{\left[E\left(c^{-2}\right)-z\right]^{-1} } & =\left(E_{0}-z\right)^{-1}-c^{-2}\left(E_{0}-z\right)^{-2} E_{1}+O\left(c^{-4}\right) \\
& =\left(E_{0}-z\right)^{-1}-c^{-2}\left(\psi_{0}, \widetilde{R}_{1}(z) \Phi_{0}\right)+O\left(c^{-4}\right),
\end{aligned}
$$

where $\Phi_{0}$ represents the eigenvector of $\widetilde{R}_{0}(z)$ to the eigenvalue $\left(E_{0}-z\right)^{-1}$ [cf., e.g., (2.19)] and

$$
\psi_{0}=\left(\begin{array}{c}
f_{0} \\
0 \\
0 \\
0
\end{array}\right]
$$

is the eigenvector of $\widetilde{R}_{0}(z)^{+}$[to the eigenvalue $\left(E_{0}-\bar{z}\right)^{-1}$ ]. Inserting $\Phi_{0}$ and $\psi_{0}$ into Eq. (2.21) and using expressions $(2.16)$ for $\widetilde{R}_{1}(z)$ finally leads to

$$
\begin{aligned}
& E_{1}=\left\{f_{0},\left[\frac{A_{1}}{2 m_{1}} v_{-} \frac{A_{1}}{2 m_{1}}+\frac{A_{2}}{2 m_{2}} v_{-} \frac{A_{2}}{2 m_{2}}+\frac{A_{1}}{2 m_{1}} w_{-} \frac{A_{2}}{2 m_{2}}+\frac{A_{2}}{2 m_{2}} w_{-} \frac{A_{1}}{2 m_{1}}\right.\right. \\
& \left.\left.\quad-E_{0} \frac{p^{2}}{4}\left[\frac{1}{m_{1}^{2}}+\frac{1}{m_{2}^{2}}\right]+2\left(m_{1}+m_{2}\right) W^{2}\right] f_{0}\right), \\
& W=\frac{w_{+}}{2\left(m_{1}+m_{2}\right)}+\frac{A_{1} A_{2}}{4 m_{1} m_{2}} .
\end{aligned}
$$

\section{Remarks}

(i) In the limit $m_{2} \rightarrow \infty$ we obtain

$$
E_{1}=\left(f_{0},\left[\frac{A_{1}}{2 m_{1}} v_{-} \frac{A_{1}}{2 m_{1}}-E_{0} \frac{p^{2}}{4 m_{1}^{2}}\right] f_{0}\right) \text {, }
$$

which is identical to our one-particle result. ${ }^{4,5}$

(ii) In practice one has to discard the term

$$
\left(f_{0}, w_{+}^{2} f_{0}\right) / 2\left(m_{1}+m_{2}\right),
$$

if the potential is determined up to first order in the coupling constant.

(iii) If $E_{0}$ is a degenerate eigenvalue of the Pauli Hamiltonian similar (but more involved) arguments apply. ${ }^{5}$

\section{DISCUSSION}

In order to compare with results obtained by completely different methods by various authors we first rewrite Eq. (2.23). Assuming $v_{ \pm}, w_{ \pm}$to be smooth enough and using the eigenvalue equation

$$
\left(\frac{p^{2}}{2 \mu}+v_{+}\right) f_{0}=E_{0} f_{0},
$$

we can eliminate $E_{0}$ in Eq. (2.23) and obtain

$$
\begin{aligned}
& E_{1}=-\left(f_{0}, \frac{p^{4}}{8} f_{0}\right)\left(\frac{1}{m_{1}^{3}}+\frac{1}{m_{2}^{3}}\right)+\epsilon_{1} \\
&+\left(\text { second-order term in } w_{+}\right) \\
& \epsilon_{1}=\left(f_{0},\left[\frac{1}{8 m_{1}^{2}}\left(2 A_{1} v_{-} A_{1}-v_{+} p^{2}-p^{2} v_{+}\right)\right.\right. \\
& \quad+\frac{1}{8 m_{2}^{2}}\left(2 A_{2} v_{-} A_{2}-v_{+} p^{2}-p^{2} v_{+}\right) \\
&+\frac{1}{4 m_{1} m_{2}}\left(A_{1} w_{-} A_{2}+A_{2} w_{-} A_{1}+A_{1} A_{2} w_{+}\right. \\
&\left.\left.\left.+w_{+} A_{1} A_{2}\right)\right] f_{0}\right)
\end{aligned}
$$

For a comparison to results in the literature it remains to determine the potential; this is trivial for scalar (and pseudoscalar) interactions and needs more comments for the vector case.

\section{A. Scalar interaction}

There we have

$$
V(r)=S(r) \beta_{1} \otimes \beta_{2}=S(r)\left(\begin{array}{cccc}
1 & 0 & 0 & 0 \\
0 & -1 & 0 & 0 \\
0 & 0 & -1 & 0 \\
0 & 0 & 0 & 1
\end{array}\right) .
$$

So we identify $v_{+}=-v_{-}=S, w_{+}=w_{-}=0$. After some straightforward algebra (assuming $S$ is smooth enough) one gets

$$
\begin{array}{r}
\epsilon_{1}=\frac{1}{8 m_{1}^{2}}\left(f_{0},[(\Delta S)+4(\nabla S) \nabla+4 S \cdot \Delta\right. \\
\left.\left.-\frac{2}{r} \frac{\partial S}{\partial r}\left(\vec{\sigma}_{1} \cdot \overrightarrow{\mathrm{x}} \times \overrightarrow{\mathrm{p}}\right)\right] f_{0}\right)+(1 \leftrightarrow 2)
\end{array}
$$

which is the same result as that of Barnes and Ghaudour; ${ }^{15}$ it disagrees with Gromes ${ }^{13}$ and with Olsson and Miller. ${ }^{14}$

\section{B. Vector interaction}

Here the identification is a bit more delicate, since there are different prescriptions for taking the instantaneous limit. It might therefore be of interest to quote the general result for the potential of Eq. (1.2), which means identifying

$$
\begin{aligned}
& v_{+}=v_{-}=v, \\
& w_{+}=w_{-}=w_{1} \vec{\sigma}_{1} \cdot \vec{\sigma}_{2}+w_{2}\left(\vec{\sigma}_{1} \cdot \hat{x}\right)\left(\vec{\sigma}_{2} \cdot \hat{x}\right) .
\end{aligned}
$$

We readily obtain from (3.2) the spin-independent $\epsilon_{1}^{\mathrm{SI}}$, the spin-orbit $\epsilon_{1}^{\mathrm{SO}}$, and the spin-spin $\epsilon_{1}^{\mathrm{SS}}$ contributions to $\epsilon_{1}=\epsilon_{1}^{\mathrm{SI}}+\epsilon_{1}^{\mathrm{SO}}+\epsilon_{1}^{\mathrm{SS}}$ : 


$$
\begin{aligned}
& \epsilon_{1}^{\mathrm{SI}}=\frac{1}{8 m_{1}^{2}}\left(f_{0}, \Delta v f_{0}\right)-\frac{1}{8 m_{1} m_{2}}\left(f_{0},\left[\left\{\left\{p_{i} w_{1} p_{i}\right\}\right\}+\left\{\left\{p_{i}\left(\hat{x}_{i} w_{2} \hat{x}_{j}\right) p_{j}\right\}\right\}\right] f_{0}\right)+(1 \leftrightarrow 2), \\
& \epsilon_{1}^{\mathrm{SO}}=\left(f_{0},\left[\frac{1}{4 m_{1}^{2}} \frac{v^{\prime}}{r}+\frac{1}{2 m_{1} m_{2}}\left[\frac{w_{2}}{r^{2}}-\frac{w_{1}^{\prime}}{r}\right]\right]\left(\vec{\sigma}_{1} \cdot \overrightarrow{\mathrm{x}} \times \overrightarrow{\mathrm{p}}\right) f_{0}\right)+(1 \leftrightarrow 2), \\
& \epsilon_{1}^{\mathrm{SS}}=\frac{1}{4 m_{1} m_{2}}\left(f_{0},\left[\left(\vec{\sigma}_{1} \cdot \vec{\sigma}_{2}\right)\left[-w_{1}^{\prime \prime}-\frac{w_{1}^{\prime}}{r}+\frac{w_{2}^{\prime}}{r}\right]+\left(\vec{\sigma}_{1} \cdot \hat{x}\right)\left(\vec{\sigma}_{2} \cdot \hat{x}\right)\left[-\frac{w_{1}^{\prime}}{r}+w_{1}^{\prime \prime}+2 \frac{w_{2}}{r^{2}}-\frac{w_{2}^{\prime}}{r}\right]\right] f_{0}\right),
\end{aligned}
$$

where we used the bracket notation of Ref. 13:

$$
\left\{\left\{p_{i} F_{i j} p_{j}\right\}\right\}=p_{i} F_{i j} p_{j}+p_{j} F_{i j} p_{i}+p_{i} p_{j} F_{i j}+F_{i j} p_{i} p_{j}
$$

and summation over repeated indices is implied.

The most direct (and safest) approach concerning the connection to a Bethe-Salpeter kernel consists now in taking

$$
V(r)=u(r)\left(\mathbb{1}_{1} \otimes \mathbb{1}_{2}-\vec{\alpha}_{1} \otimes \vec{\alpha}_{2}\right)
$$

which means one neglects the $q_{0}$ dependence of $\widetilde{u}\left(-q_{0}{ }^{2}+\vec{q}^{2}\right)$ in the kernel. Then one identifies

$$
v=u, w_{1}=-u, w_{2}=0 ;
$$

this yields agreement of $\epsilon_{1}^{\text {SO }}$ and $\epsilon_{1}^{\text {SS }}$ with Refs. 13 and 20; $\epsilon_{1}^{\text {SI }}$ differs.

If an expansion of $\tilde{u}\left(-q_{0}{ }^{2}+\overrightarrow{\mathrm{q}}^{2}\right) \simeq \tilde{u}\left(\overrightarrow{\mathrm{q}}^{2}\right)-q_{0}{ }^{2} \tilde{u}^{\prime}\left(\overrightarrow{\mathrm{q}}^{2}\right)$ is allowed one may use current conservation to transform the second term and obtain

$$
v=u, w_{1}=-\frac{u}{2}, \quad w_{2}=\frac{r}{2} u^{\prime} .
$$

Now all three contributions in (3.6) agree with Ref. 13.
It is interesting to note that both the spin-orbit and the spin-spin interaction terms are insensitive to the different limit descriptions. This is so because both expressions depend only on the sum $-w_{1}^{\prime}+w_{2} / r$, which is equal to $u^{\prime}$ in both cases.

So let us finally conclude that we have given an unambiguous scheme to extract relativistic corrections to bound-state energies of two-particle systems if a timeindependent potential is given; the step from a relativistic wave equation to the Hamiltonian description depends on the procedure one chooses.

\section{ACKNOWLEDGMENTS}

F. G. and H. G. would like to thank the members of the Zentrum für Interdisziplinäre Forschung der Universität Bielefeld and the CERN-Theory Group, respectively, for their warm hospitality, and especially T. Fulton, V. Glaser, H. Leutwyler, A. Martin, and L. Streit for their interest. Financial support by the Alexander von Humboldt Foundation (F.G.) is gratefully acknowledged. B. T. was supported by Fonds zur Förderung der Wissenschaftlichen Forschung in Österreich, project Nr 3764.
*On leave of absence from Institut für Theoretische Physik, Universität Graz, Austria.

${ }^{\dagger}$ Permanent address: Institut für Theoretische Physik, Universität Wien, Austria.

${ }^{1}$ L. L. Foldy and S. A. Wouthuysen, Phys. Rev. 78, 29 (1950).

${ }^{2}$ F. Gesztesy, H. Grosse, and B. Thaller, Phys. Lett. 116B, 155 (1982).

${ }^{3}$ F. Gesztesy, H. Grosse, and B. Thaller, Adv. Appl. Math. (to be published).

${ }^{4}$ F. Gesztesy, H. Grosse, and B. Thaller, Phys. Rev. Lett. 50, 625 (1983).

${ }^{5}$ F. Gesztesy, H. Grosse, and B. Thaller, Ann. Inst. Henri Poincaré A 40, 159 (1984).

${ }^{6}$ H. A. Bethe and E. E. Salpeter, Quantum Mechanics of Oneand Two-Electron Atoms (Academic, New York, 1959).

${ }^{7}$ T. P. Das, Relativistic Quantum Mechanics of Electrons (Harper and Row, New York, 1973).

${ }^{8}$ D. Flamm and F. Schöberl, Introduction to the Quark Model of
Elementary Particles (Gordon and Breach, New York, 1982), Vol. I.

${ }^{9}$ T. Appelquist, R. M. Barnett, and K. D. Lane, Annu. Rev. Nucl. Sci. 28, 387 (1978).

${ }^{10} \mathrm{M}$. Krammer and H. Krasemann, in New Phenomena in Lepton-Hadron Physics, edited by D. Fries and J. Wess (Plenum, New York, 1979).

${ }^{11}$ O. Steinmann, Nucl. Phys. B196, 394 (1982).

12P. Moxhay and J. L. Rosner, Phys. Rev. D 28, 1132 (1983).

13D. Gromes, Nucl. Phys. B131, 80 (1977).

${ }^{14}$ M. G. Olsson and K. J. Miller, Phys. Rev. D 28, 674 (1983).

${ }^{15}$ T. Barnes and G. I. Ghandour, Phys. Lett. 118B, 411 (1982).

${ }^{16}$ E. Eichten and F. Feinberg, Phys. Rev. Lett. 43, 1205 (1979).

${ }^{17}$ E. Eichten and F. Feinberg, Phys. Rev. D 23, 2724 (1981).

${ }^{18}$ W. Buchmüller, Phys. Lett. 112B, 479 (1982).

${ }^{19}$ D. Gromes, Z. Phys. C 18, 249 (1983).

${ }^{20}$ H. J. Schnitzer, Phys. Rev. D 13, 74 (1976). 